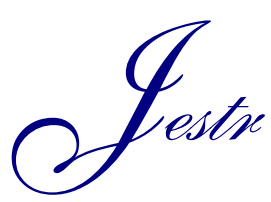

\title{
Urban Change Detection in TerraSAR Image Using the Difference Method and SAR Coherence Coefficient
}

\author{
Zhang Xuedong ${ }^{1,2}$, Liu Wenxi ${ }^{1,2}$ and He Shuguang ${ }^{3, *}$ \\ ${ }^{1}$ Beijing University of Civil Engineering and Architecture, Beijing 102616, China \\ ${ }^{2}$ Key Laboratory of modern urban surveying and mapping, State Bureau of surveying, mapping and Geographical Information Bureau, \\ Beijing 102616, China \\ ${ }^{3}$ China Association of Construction Education, Beijing 100037, China
}

Received 20 December 2017; Accepted 14 May 2018

\begin{abstract}
At present, most methods of urban change detection with synthetic aperture radar (SAR) images are based on image amplitude information. SAR images of the same region over different periods are usually compared and analyzed by using the difference method, which often neglects the SAR image interference information, exhibiting sensitivity to changes in ground scatterers. Therefore, this study presented a novel method combining the difference method and the SAR coherence coefficient to accurately and comprehensively detect the characteristics of urban change information. In this method, the amplitude difference between two images was obtained by the image difference method, and the suspected change areas in the urban area were determined. The suspected change areas of the image were further analyzed by using the coherence coefficient of the images and were regarded as genuinely changed areas when the results of the difference method and the coherence coefficient both changed. Finally, the proposed method and the difference method were employed to conduct a comparative study at Huilongguan Village in Changping District of Beijing city, China. Results indicate that the difference method can only reflect urban changes caused by urban construction (e.g., demolition and urban expansion), whereas the proposed method can not only detect urban changes due to urban construction but also remove urban pseudo-changes attributed to season change, vegetation, and tree growth. Thus, the proposed method can effectively improve the accuracy of change detection. Comparative analysis and field verification of the selected region of interest showed that the result accuracy of the proposed method reaches $84.5 \%$, which is much higher than that of the single difference method $(61.4 \%)$. This finding proves that the proposed method is feasible for urban change detection, and the conclusions can also provide a reference for urban planning and construction.
\end{abstract}

Keywords: TerraSAR image, Difference method, Coherence coefficient, Urban change detection

\section{Introduction}

Synthetic Aperture Radar (SAR) has the characteristics of fixed revisit period, all-day and all-weather operation, and the capability to penetrate most objects, all of which make it more suitable for detecting change information compared with optical remote sensing [1]. With the development of radar remote sensing technology, various types of spaceborne SAR system have been put into operation, and a variety of high-resolution SAR images have been obtained, providing massive data resources for SAR for urban change detection. However, the urban change detection methods based on SAR images are far from able to meet actual needs $[1,2]$. Therefore, the study of change detection methods based on SAR images has become a hot topic of current concern.

Dynamic detection of radar images is a type of means to quantitatively analyze and determine the features and processes of surface change from the image data of different periods, and these methods mostly deal with multi-phase radar images [1-5]. These methods involve several

\footnotetext{
*E-mail address: 44047461@qq.com

ISSN: $1791-2377$ @ 2018 Eastern Macedonia and Thrace Institute of Technology. All rights reserved. doi:10.25103/jestr.113.03
}

parameters, such as type of change, distribution, variable quantity, ground type, boundary, and change trend [3-5], which can provide the spatial distribution of the ground feature and the qualitative and quantitative information of the change. At present, the study of urban change detection using SAR data is mainly based on the amplitude information of SAR images [1-8]. The change area of land surface property is also extracted by the difference method to compare and analyze the SAR images of the same region at different periods. Zhang et al. used the difference method to detect land change with high-resolution TerraSAR-X images, and their results could reflect the information of newly increased buildings; however, the disadvantage of their method was that the interference information between images was not considered, resulting in insufficient accuracy of change detection [3]. Some studies showed that the coherence coefficient of SAR images was related to the different scattering characteristics of the objects in the image of two different phases [9-12]. Moreover, coherence coefficient was sensitive to the change of scattering characteristics of the objects, which had a key value for the detection of urban change information. Therefore, considering the amplitude and interference information of SAR images, the image difference method and SAR coherence coefficient were combined to obtain accurate and comprehensive change information of urban area. 


\section{State of the art}

At present, studies on SAR image change information extraction mainly focus on two aspects, namely, amplitude information [1-8] and interference information of SAR images [9-12]. The change detection method based on the amplitude information of SAR images can be divided into three categories: image difference method, postclassification comparison method, and direct classification method [2], among which the image difference method is the most common one [2]. Chen et al. systematically introduced the algorithm commonly used in SAR change detection and the core decision rule in SAR image change detection from a pre-processing algorithm; the processing algorithm and its performance were discussed and evaluated to a certain degree after the verification [2]. Cao et al. used the improved Dempster-Shafer algorithm to detect the change information of urban areas by combining the features of SAR images at different time intervals [4]. Wang et al. employed the logarithmic ratio method to construct a difference image and then used the Markov random field model to extract the land change area of urban buildings; however, this method could not meet the need for fine change detection in urban areas because of noise and image brightness [1]. Gamba et al. conducted a detection study of buildings by using airborne high-resolution SAR images [5-7]. Brenner analyzed the potential of ultra-high-resolution SAR and InSAR data for urban detection and discussed the effects of different combinations of vision on the detection of urban targets [8]. The fusion of different-vision SAR data result was another difficult problem to solve. The urban change extraction method based on SAR interference information mainly used the sensitivity of the coherence coefficient image to detect the scattering change of ground objects. Zhang et al. extracted the outermost boundary of a city with the high coherence of a stable urban surface [10]. Wang et al. classified urban and construction land, cultivated land and water body by using the high and low coherence characteristics of different objects [11]. Pulvirenti et al. successfully extracted the change of urban flood waterline with SAR coherence coefficient; however, determining how to perform the flood mapping algorithm with SAR coherence coefficient needed further study [13]. Ma et al. effectively removed a mining subsidence area with a SAR coherence coefficient image and realized the rapid assessment of surface subsidence caused by high-intensity mining activity. However, obtaining the boundary of the subsidence area with the coherence coefficient image was difficult when the study area was large [14]. In these methods, only the amplitude information of the SAR image was considered or only the interference information of the SAR image was used.

Change information detection in an urban area based on SAR images must consider the amplitude difference between the change information and the background value as well as the sensitivity of the interference information to the change of the ground objects' scattering characteristics. However, the preceding methods often cannot consider the two aspects, resulting in insufficient accuracy of the extracted urban change information. Therefore, a test area in Beijing was taken as an example by using the high-resolution TerraSAR image. Considering the amplitude and interference information of SAR images, this study combined the difference method and the coherence coefficient to analyze the change information of urban areas. The change information of buildings in the test area was successfully extracted, and the detection accuracy of the proposed method was verified. The study results can provide a reference for urban planning and construction.

The remainder of this study is organized as follows: Section 3 describes the algorithm of the image difference method and the coherence coefficient, and proposes an urban change detection method based on the coherence coefficient and the difference method. It also provides the accuracy assessment index of change detection. Section 4 analyzes the urban change information with the proposed method in a test area. The rationality and feasibility of the proposed method are verified through comparative analysis and field investigation. Section 5 summarizes this study and provides relevant conclusions.

\section{Methodology}

\subsection{Image difference method}

Image difference method is a change detection method that subtracts or divides the radar images of two phases. Its principle is that the land class does not change in the radar images of two phases, which generally have equal or similar amplitude values, whereas the local land class changes and the amplitude value of the corresponding location have a large difference. Therefore, the difference between the amplitude value in the land-class change area and the background value will be large, and the difference image will be post-processed to detect the change information of the feature in the test area. The mathematical expression of the method is shown in Equation (1) and Equation (2).

$$
\begin{aligned}
& \Delta B=B_{T_{2}}-B_{T_{1}} \\
& \Delta B=B_{T_{2}} / B_{T_{1}}
\end{aligned}
$$

Where $\Delta B$ denotes the difference image, $B_{T_{1}}$ and $B_{T_{2}}$ denotes the image of the time-phase $T_{1}$ and $T_{2}$, respectively.

\subsection{Coherence coefficient}

The coherence coefficient is a crucial value in InSAR processing. The coherence coefficient of the complex images is used to measure the similarity between images and the quality of interference interferogram, which provides important information on the ground scatterers [15-18]. The coherence coefficient of images $s_{1}$ and $s_{2}$ can be defined as shown in Equation (3).

$$
\rho=\frac{\sum\left|s_{1}(j, k) \times s_{2}^{*}(j, k)\right|}{\sqrt{\sum\left|s_{1}(j, k) \times s_{1}^{*}(j, k)\right| \times \sum\left|s_{2}(j, k) \times s_{2}^{*}(j, k)\right|}}
$$

Where $*$ denotes a conjugate complex, $s_{1}$ and $s_{2}$ are prefiltered single-look complex SAR images, and $\rho$ is the coherence coefficient whose value is between 0 and $1(0$ denotes decoherence and 1 denotes complete coherence). The factors that generally affect coherence are spatial baseline, time baseline, Doppler center shift, complex image registration, and system thermal noise. During the processing of TerraSAR-X images, the two-phase images are precisely registered to eliminate the decoherence caused by the registration. The range and azimuth of the image are filtered to remove the Doppler center shift in the azimuth 
direction and the decoherence caused by the spatial baseline. After these processes, the coherence coefficient, which is sensitive to the scatter characteristics of the SAR image, is mainly related to the variation of the ground objects' scattering characteristics in the two phases of image acquisition [12]. Therefore, the coherence coefficient is crucial for the detection of urban change information [12-14].

\subsection{Combination of image difference method and coherence coefficient}

This study combined the image difference method based on the amplitude information with the coherence coefficient of SAR images to detect the urban change. On the basis of the SAR images of different phases in the same region, this method involves a series of steps, such as data preprocessing, image correction, difference processing, difference image, coherent image overlay, and border processing. Fig. 1 shows the specific processing flow.

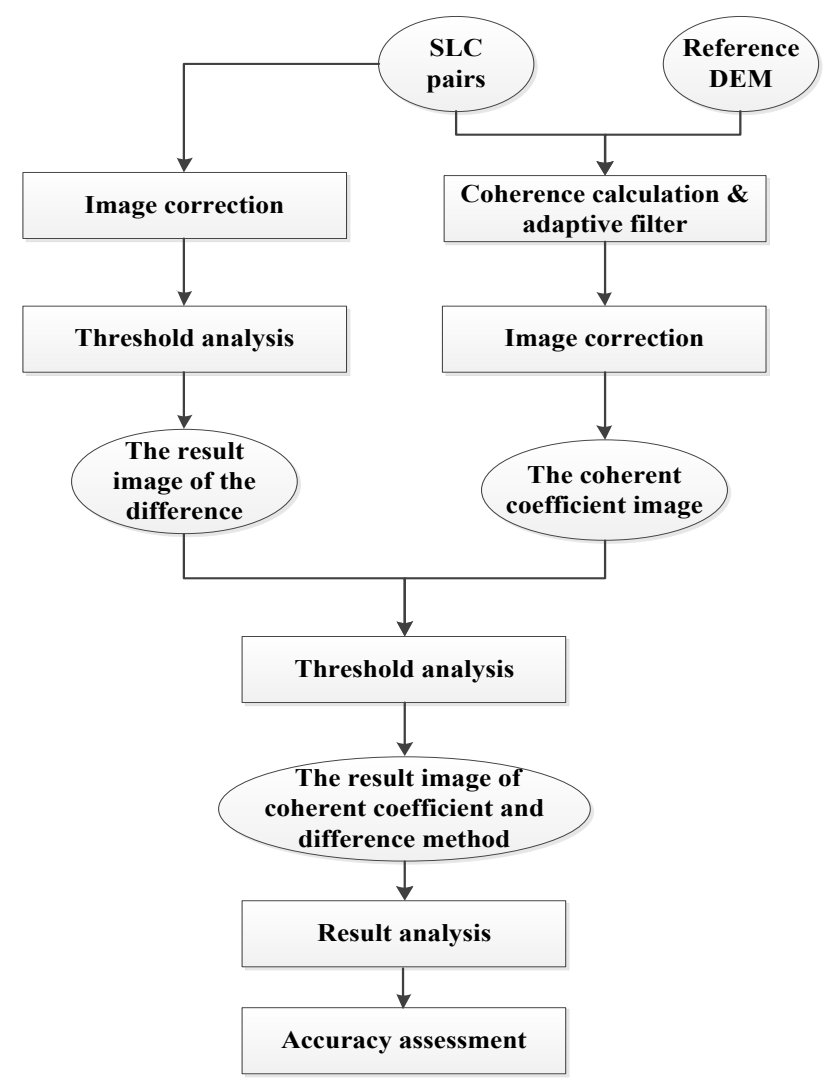

Fig. 1. Data processing flow

The registration has two types: one is the registration between the SAR images of two phases, and the other is the registration of the SAR image with the generated coherence image. Given that the SAR images and the coherence images are all radar coordinates, the radar coordinate must be converted to the geodetic coordinate system. The identification points are usually chosen by humans in this process. The projection transformation equation is established according to the polynomial fitting method, and the projection relationship and the data resampling are realized according to the polynomial. Finally, sub-pixel auto-fine registration is achieved via polynomial registration $[2,3]$.

\subsection{Assessment of detection accuracy}

Visual interpretation is the most intuitive method for determining the reliability of change detection. However, it only assesses the validity of the change detection method from a qualitative perspective. A quantitative method is required to verify the effectiveness of the proposed method for an accurate evaluation of the change detection information. The accuracy of change detection is quantitatively evaluated in this study by using the percentage correct classification (PCC). The specific evaluation index expression is shown in Equation (4).

$$
P C C=(T P+T N) /(T P+T N+F P+F N)
$$

Where $T P$ indicates the number of pixels that have changed and have been detected, $T N$ indicates the number of pixels that remained unchanged and have not been detected, FP indicates the number of pixels that have remained unchanged but have been detected as being changed, and $F N$ indicates the number of pixels that have changed but have not been detected.

\section{Result analysis and discussion}

\subsection{Test data}

The TerraSAR-X images of two phases in Beijing city of China with the $\mathrm{HH}$ polarization mode were adopted as the test data in this study. The range resolution is $2.15 \mathrm{~m}$, the azimuth resolution is $3.3 \mathrm{~m}$, and the software used was EnviSARcape and MATLAB. The specific parameters of the SAR image are shown in Table 1.

Huilongguan Village of Changping District in Beijing city was selected as the test area. The image size was 830 rows $\times 1150$ columns. The two images were acquired in November 2011 and February 2013. In the two periods, the season of rare vegetation in Beijing avoided the decoherence phenomenon caused by the vegetation growth to a large extent. Fig. 2 and 3 show the SAR amplitude images of two phases and the coherence coefficient image of the two SAR images, respectively.

Table 1. Data parameters of TerraSAR-X

\begin{tabular}{|c|c|c|c|c|c|}
\hline Number & Acquisition time & close distance (m) & center slant distance (m) & long distance (m) & incidence angle $\left({ }^{\circ}\right)$ \\
\hline $\begin{array}{l}1 \\
2\end{array}$ & $\begin{array}{l}20111128 \\
20130119\end{array}$ & $\begin{array}{l}594405.31084829604 \\
595496.61022556713\end{array}$ & $\begin{array}{l}603636.66549329821 \\
604371.47895854013\end{array}$ & $\begin{array}{l}612867.11073482828 \\
613245.43828801555\end{array}$ & $\begin{array}{l}33.128361880663498 \\
33.203756051081349\end{array}$ \\
\hline
\end{tabular}


Zhang Xuedong, Liu Wenxi and He Shuguang/

Journal of Engineering Science and Technology Review 11 (3) (2018) 18-23

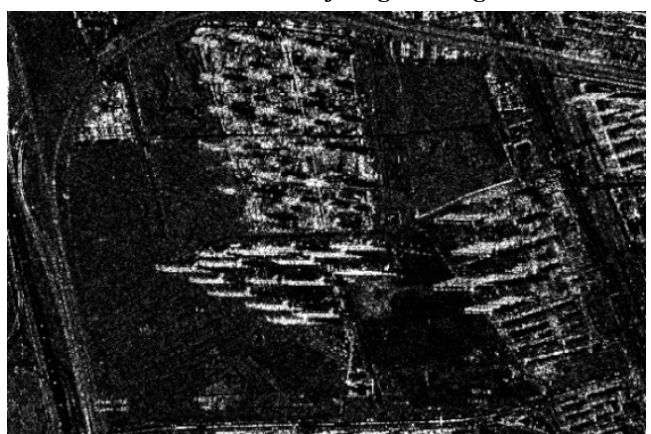

(a) SAR image on November 28, 2011

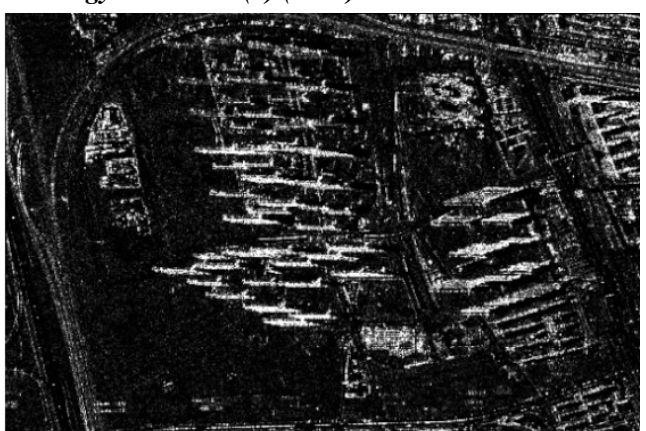

(b) SAR image on February 21, 2013

Fig. 2. SAR amplitude images of two phases

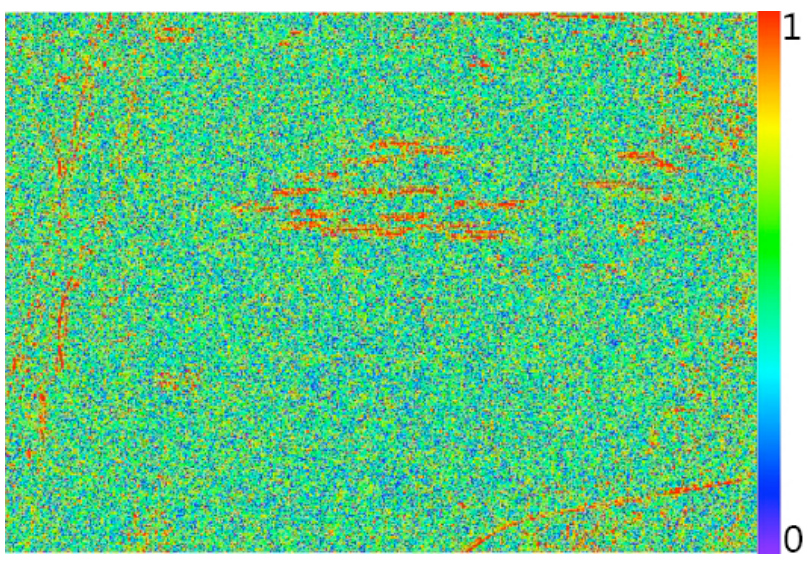

Fig. 3. Coherence coefficient image

\subsection{Result analysis of the image difference method}

The difference value between two amplitude images can be obtained by subtracting the two-phase SAR amplitude images with the difference method. Threshold choice is important for the difference method. The average of the calculated amplitude gradient of the entire image was used as the threshold for accuracy. The change areas generated by the difference method were often discretely distributed point targets. These discrete point targets in the difference image must be merged into a planar target to reconstruct the geometric outline of the change area [3]. Fig. 4 shows the overlay image of the difference result between the two amplitude images obtained by using the difference method and the original image. The red circle area in the figure depicts the change information detected by the single difference method.

The area of the Lantianjiayuan community (Fig. 5), whose size is 260 rows $\times 170$ columns, was selected as the region of interest in the test to evaluate the accuracy of detection results with the difference method. The detection results are shown in Table 2 .

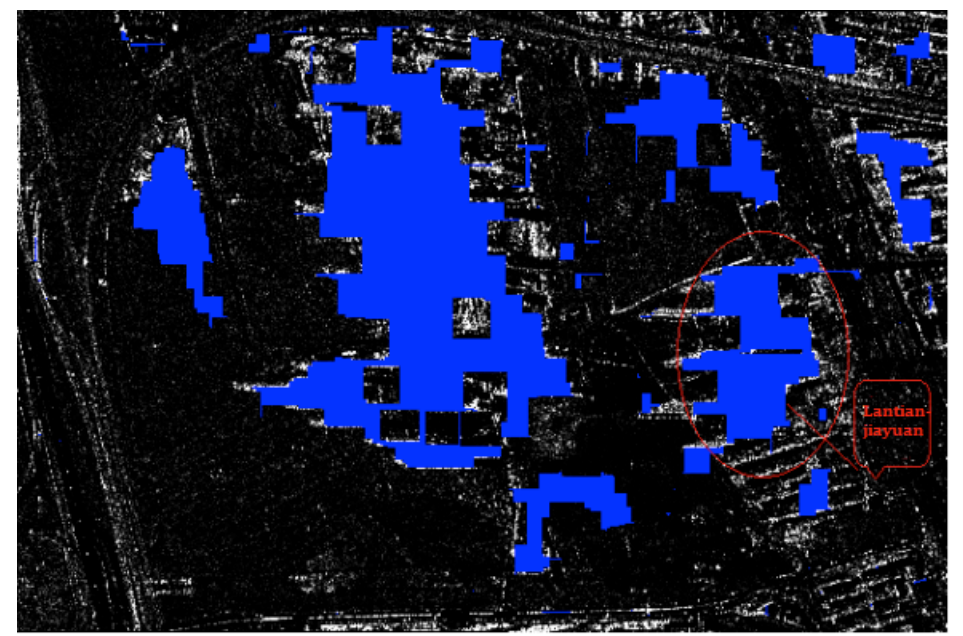

Fig. 4. Result image of the difference method

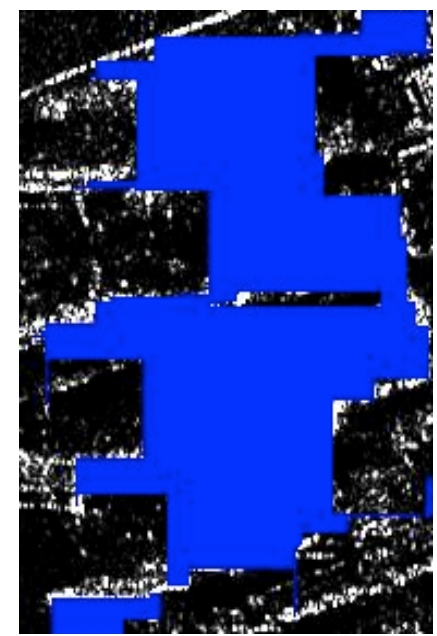

Fig. 5. Lantianjiayuan community

\begin{tabular}{l|l} 
Table 2. Detection accuracy verification table (the difference method) \\
\hline Accuracy index & Number \\
\hline TP & 7282 \\
TN & 19848 \\
FP & 12206 \\
FN & 4864 \\
\hline
\end{tabular}


According to Equation (4), the PCC of the difference method is $61.4 \%$. Therefore, a certain degree of false detection still exists when a single difference method is used After analysis, the reason for urban change mainly includes two aspects. One aspect is the change of the ground objects' scattering properties due to the change caused by the demolition and the expansion of the city. The dielectric coefficient also changes with the scattering properties, and the amplitude value in the SAR image will change considerably. The other aspect is the change in amplitude value of the SAR image due to the change of seasons and the growth of vegetation and trees; however, these changes are not real urban changes. Therefore, identification and removal of such changes in the test area are essential. Although tree growth and season change will cause a change in the structure, the position of the scattering elements, and the amplitude value of the SAR image, the scatterers' structure will not change and the coherence will remain high in the SAR coherence image. Therefore, the changes caused by the tree growth and season change can be removed.

\subsection{Result analysis of the proposed method}

The result images obtained by the difference method were overlaid on the coherence coefficient image generated by the two-phase SAR images, and the change area was then further analyzed according to the coherence. When the result image of the difference method changed and the coherence image didn't change, the area was unchanged. The area changed only when the coherence coefficient image and the result image of the difference method both changed. This study first conducted sample selection in the coherence coefficient image to select a suitable coherence coefficient as a threshold and remove the change due to vegetation growth and season change. Typical samples of buildings, vegetation, and roads in different locations in the coherence coefficient image were selected, and the sample mean was calculated as the coherence coefficient of the corresponding objects. The results are shown in Table 3. The coherence coefficients of different objects could be roughly determined, and a suitable coherence coefficient was obtained as a threshold for analysis through repeated experiments. Finally, 0.115 was chosen as the threshold. On the basis of this threshold, the change area in the difference images owing to vegetation growth and season change was removed. Fig. 6 shows the change area image gained by overlaying the difference of the result image and the coherence coefficient image.

Lantianjiayuan community was taken as the test object to evaluate the detection accuracy by combining the difference method and the coherence coefficient. The detection result is shown in Table 4.

Table. 3. Average coherent coefficients of different ground features

\begin{tabular}{l|l|l|l}
\hline $\begin{array}{l}\text { The category of ground } \\
\text { feature }\end{array}$ & Building & Road & Vegetation \\
\hline $\begin{array}{l}\text { The chosen points } \\
\begin{array}{l}\text { The average of coherent } \\
\text { coefficient }\end{array}\end{array}$ & 35 & 18 & 32 \\
\hline
\end{tabular}

Table. 4. Detection accuracy verification table (the proposed method)

\begin{tabular}{c|c} 
Table. 4. Detection accuracy verification table (the proposed method) \\
\hline Accuracy Index & Number \\
\hline TP & 7282 \\
TN & 30048 \\
FP & 2186 \\
FN & 4684 \\
\hline
\end{tabular}

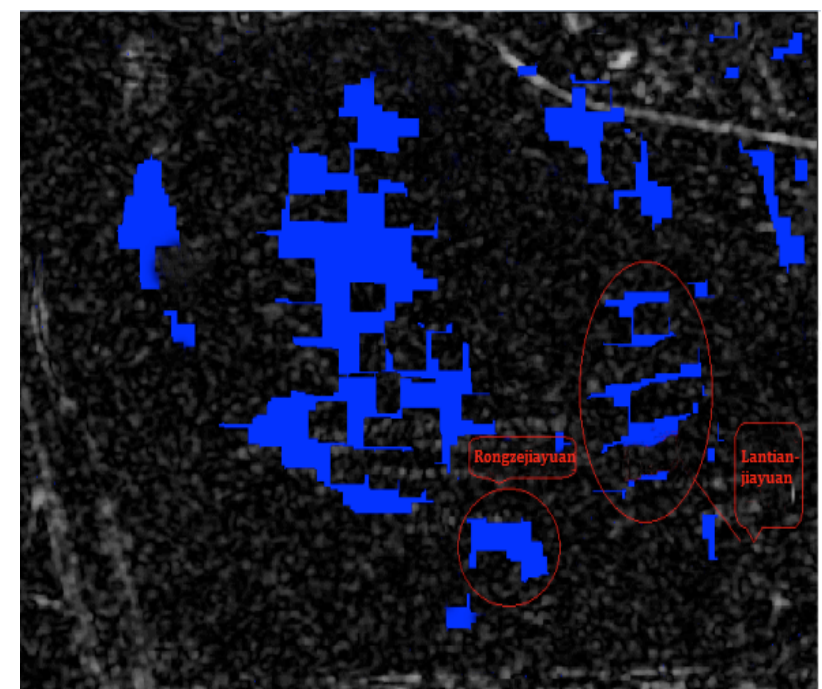

Fig. 6. Result image of the proposed method

According to Equation (4), the PCC of the proposed method is $84.5 \%$. The detection accuracy of the proposed method improved considerably compared with that of the single difference method. Fig. 7 shows the detection result obtained by combining the difference method and the coherence coefficient. A comparison of Fig. 6 and 7 showed that part of the change area was removed because of vegetation growth and season change. The field investigation found that the removed areas were the green areas of Lantianjiayuan community, which were indeed vegetation, thereby verifying the accuracy of the test result. A comparison of the change area with the land planning map

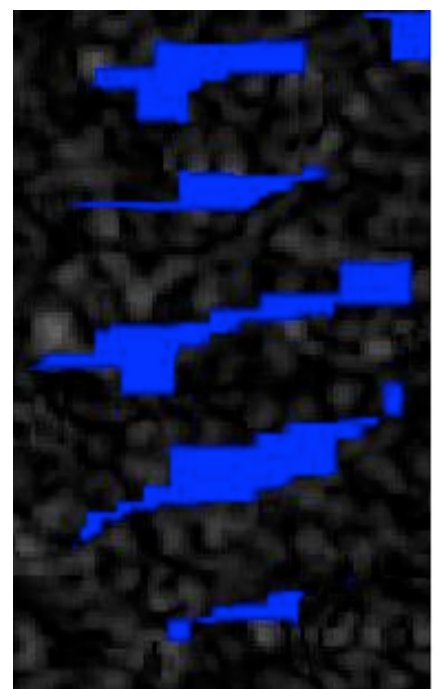

Fig. 7. Lantianjiayuan community

of Changping District in 2012 and a field study conducted on the newly built buildings in the change area show that the change area extracted by the proposed method was consistent with the information obtained from the land planning map. However, the detection result was affected by the SAR side-view imaging mode, the image acquisition angle, and the reflection of scatterers in the test to some extent. Change information in the layover region cannot be effectively detected owing to the SAR side-view imaging mode. Therefore, determining how to solve change information detection in the layover region is worth studying. 


\section{Conclusions}

The method combining the difference method with the coherence coefficient was proposed to accurately and comprehensively detect urban change information. A study on the change information of the urban area was conducted based on the high-resolution image of TerraSAR. The following conclusions were obtained:

(1) A detection method based on the difference method and SAR coherence coefficient, which combines the amplitude and the interference information of the SAR image, was proposed.

(2) The proposed method can detect urban change information caused by urban construction (e.g., demolition and urban expansion) and remove urban pseudo-change due to season change, vegetation, and tree growth. The detection accuracy of change information can be improved considerably, and the correct detection rate can reach $84.5 \%$.

(3) The change situation of Huilongguan Village of Changping District in Beijing was successfully extracted by the proposed method, proving that the change situation is consistent with the land planning information of Changping District in 2012. Results show the feasibility of using the proposed method to detect urban change.
The proposed method considers the amplitude and coherence of SAR images, which can accurately detect real urban change information. It also provides a technical reference for detecting urban change information based on SAR images. However, the selection of the coherence coefficient threshold is affected by the human experience and the SAR imaging mode. These factors cause a slight deviation of the extracted information of urban change from the real change. Therefore, determining the rational selection of the coherence coefficient threshold and integrating the SAR data of ascending and descending orbits to detect change information will become the focus of the future study.

\section{Acknowledgements}

The study was supported by the Scientific Study Project of Beijing Educational Committee (No. SQKM201710016009), Fundamental Research Funds for Beijing University of Civil Engineering and Architecture (No. X18048), and Beijing University of Civil Engineering and Architecture Basic Study Fund (No. KYJJ2017025).

This is an Open Access article distributed under the terms of the Creative Commons Attribution Licence

\section{References}

1. Wang W. H., Jiang T., Zhang Y. H., Kang Y. H., "Detection of urban land use changes based on high resolution SAR images". Geomatics \& Spatial Information Technology, 39(7), 2016, pp.141144.

2. Chen F. L., Zhang H., Wang C., "The art in SAR change detection-a systematic review". Remote Sensing Technology and Application, 22(1), 2007, pp.109-115.

3. Zhang R., Liu G. X., Li T., Yu B., Xu Z., "Detection of urban land use changes based on high resolution TerraSAR-X images". Railway Investigation and Surveying, 37(4), 2011, pp.17-20.

4. Cao G. Z., Hou P., Jin Y. Q., Mao X. Q., "Fusion of features in multi-temporal SAR imagery to detect changes in urban areas". International Journal of Remote Sensing, 30(22), 2009, pp.59896001.

5. Gamba P., Houshmand B., Saccani M., "Detection and extraction of buildings from interferometric SAR data". IEEE Transactions on Geoscience and Remote Sensing, 38(1), 2000, pp.611-617.

6. Ciuonzo D., Carotenuto V., Maio A. D., "On Multiple Covariance Equality Testing with Application to SAR Change Detection". IEEE Transactions on Signal Processing, 65(19), 2017, pp.5078 5091.

7. Gong, M., Zhao, J., Liu, J., Miao, Q., \& Jiao, L., “Change Detection in Synthetic Aperture Radar Images Based on Deep Neural Networks". IEEE Transactions on Neural Networks \& Learning Systems, 27(1), 2017, pp.125-138.

8. Brenner A. R., Roessing L., "Radar imaging of urban areas by means of very high-resolution SAR and interferometric SAR". IEEE Transactions on Geoscience and Remote Sensing, 46(10), 2008, pp.2971-2982.

9. Perissin D., Wang T., "Time-series InSAR applications over urban areas in China". IEEE Journal of Selected Topics in Applied Earth Observations and Remote Sensing, 4(1), 2011, pp.92-100.
10. Zhang W. Y., Liu Q. C., Meng X. J., "City boundary extraction based on interferometric coherent Image". Geomatics \& Spatial Information Technology, 37(5), 2014, pp.56-59.

11. Wang X. S., Chen E. X., Li Z. Y., Yao W. Q., Zhao L., "Multitemporal and Dual-Polarization Interferometric SAR for land cover tyge classification". Acta Geodaetica et Cartographica Sinica, 44(5), 2015, pp.533-540.

12. Hooper A., "A multi-temporal InSAR method incorporating both persistent scatterer and small baseline approaches". Geophysical Study Letters, 35(16), 2008, pp.96-106.

13. Pulvirenti L., Chini M., Pierdicca N., Boni G., "Use of SAR data for detecting floodwater in urban and agricultural areas: the role of the interferometric coherence". IEEE Transactions on Geoscience and Remote Sensing, 54(3), 2016, pp.1532-1544.

14. Ma W., Ma C., Zhao P. F., Zhang W. Y., "Extraction of mining subsidence area based on coherence image of high resolution SAR". Science of Surveying and Mapping, 43 (5), 2017, pp.56-65.

15. Inglada J., Mercier G., "A new statistical similarity measure for change detection in multitemporal SAR images and its extension to multiscale change analysis". IEEE Transactions on Geoscience and Remote Sensing, 45(5), 2007, pp.1432-1445.

16. Pham M. T., Mercier G., Michel J., "Change detection between SAR images using a pointwise approach and graph theory". IEEE Transactions on Geoscience and Remote Sensing, 54(4), 2016, pp.2020 - 2032.

17. Li Z.F., Bao Z., Li H., Liao G. S., "Image autocoregistration and InSAR interferogram estimation using joint subspace projection". IEEE Transactions on Geoscience and Remote Sensing, 44(2), 2006, pp.288-297.

18. Hyde P., Dubayah R., Walker W., Blair, J. B., Hofton, M. \& Hunsaker, C., "Mapping forest structure for wildlife habitat analysis using multi-sensor (LiDAR, SAR/InSAR, ETM+, Quickbird) synergy". Remote Sensing of Environment, 102(1-2), 2006, pp.63-73. 